\title{
Hybrid valve-in-valve mitral valve replacement
}

\author{
Morgan T. Harloff, MD, ${ }^{\mathrm{a}}$ Andrew R. Papoy, MD, ${ }^{\mathrm{a}}$ Ayaz Aghayev, MD, ${ }^{\mathrm{b}}$ and Tsuyoshi Kaneko, MD, ${ }^{\mathrm{a}}$ \\ Boston, Mass
}

From the ${ }^{\mathrm{a} D i v i s i o n}$ of Cardiac Surgery, and ${ }^{\mathrm{b}}$ Noninvasive Cardiovascular Imaging Section, Department of Radiology, Brigham and Women's Hospital, Harvard Medical School, Boston, Mass.

Disclosures: Dr Kaneko reported speaker for Edwards Lifesciences. All other authors reported no conflicts of interest.

The Journal policy requires editors and reviewers to disclose conflicts of interest and to decline handling or reviewing manuscripts for which they may have a conflict of interest. The editors and reviewers of this article have no conflicts of interest.

This study was approved by the institutional review board of Brigham and Women's Hospital (protocol number 2010P000292). Patient consent was waived by the institutional review board for this study.

Accepted for the 100th Annual Meeting of The American Association for Thoracic Surgery.

Received for publication April 21, 2020; revisions received April 21, 2020; accepted for publication May 8, 2020; available ahead of print June 25, 2020.

Address for reprints: Tsuyoshi Kaneko, MD, Division of Cardiac Surgery, Brigham and Women's Hospital, 75 Francis St, Boston, MA 02115 (E-mail: tkaneko2@partners.org).

JTCVS Techniques 2020;3:154-6

2666-2507

Copyright (C) 2020 The Authors. Published by Elsevier Inc. on behalf of The American Association for Thoracic Surgery. This is an open access article under the CC BY-NC-ND license (http://creativecommons.org/licenses/bync-nd/4.0/).

https://doi.org/10.1016/j.xjtc.2020.05.032

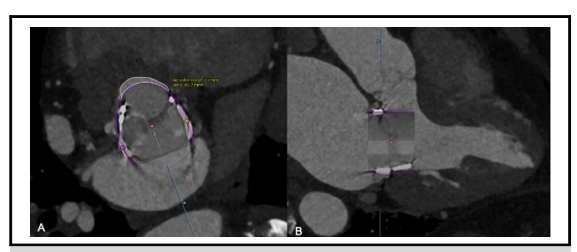

A, Predicted neo-LVOT area was $0.46 \mathrm{~cm}^{2}$. B, ViVTMVR would have caused LVOT obliteration.

CENTRAL MESSAGE

Hybrid valve-in-valve mitral valve replacement (MVR) may add another option to address the problem of left ventricular outflow tract obstruction in high-risk patients for whom repeat MVR is best avoided.

See Commentaries on pages 157,158 , and 160.
Video clip is available online.

Valve-in-valve (ViV) transcatheter mitral valve replacement (TMVR) using an Edwards SAPIEN 3 valve (Edwards Lifesciences, Irvine, Calif) has emerged as an alternative for patients at prohibitive risk with reoperation. ${ }^{1,2}$ However, iatrogenic left ventricular outflow tract (LVOT) obstruction is a potentially life-threatening complication associated with this technique. ${ }^{3}$ ViV-TMVR induced LVOT obstruction occurs when the transcatheter valve metal frame pushes the anterior leaflet of a bioprosthesis toward the interventricular septum, consequently narrowing the LVOT. ${ }^{3}$ Hemodynamic instability results due to impediment of blood flow across the LVOT during systole. ${ }^{3}$

Preoperative computed tomography-based simulation can identify patients at increased anatomical risk for LVOT obstruction. ${ }^{3}$ Predicted neo-LVOT area $\leq 1.7 \mathrm{~cm}^{2}$ carries high sensitivity and specificity for postprocedural LVOT obstruction. ${ }^{3}$ We report a case in which a patient at high risk for redo mitral valve replacement (MVR) and LVOT obstruction with ViVTMVR instead underwent a hybrid transatrial approach with cardiopulmonary bypass (CBP). This technique allows for resection of previously placed bioprosthetic leaflets and reduces the risk of neo-LVOT obstruction posed by ViV-TMVR.

\section{CLINICAL SUMMARY}

A 71-year-old female patient with severe bioprosthetic mitral stenosis was evaluated by the structural heart team. Nine years previously, she underwent MVR with a 29-mm Edwards Magna Ease bioprosthetic valve (Edwards Lifesciences) via minimally invasive right thoracotomy. Her operation was complicated by failed repair necessitating valve replacement. The initial operation and follow-up occurred at the referring hospital.

Her Society of Thoracic Surgeons score was 3.5\%. However, her Charlson Comorbidity Index of 6 and Katz Index of 2 of 6 indicated she was frail, deeming her high risk for reoperative MVR. She was considered for ViV-TMVR but preoperative TMVR computed tomography revealed a projected neo-LVOT area of $0.46 \mathrm{~cm}^{2}$ with a 29 -mm SAPIEN 3 (Figure 1, A). ViV-TMVR would have resulted in near-total obliteration of her LVOT at end-systole (Figure 1,B). Due to her high-risk nature and concerns for prolonged cardiopulmonary bypass (CPB) required for repeat MVR, we elected to proceed with a hybrid approach via median sternotomy that allowed for resection of the anterior bioprosthetic leaflets before ViV-MVR. 


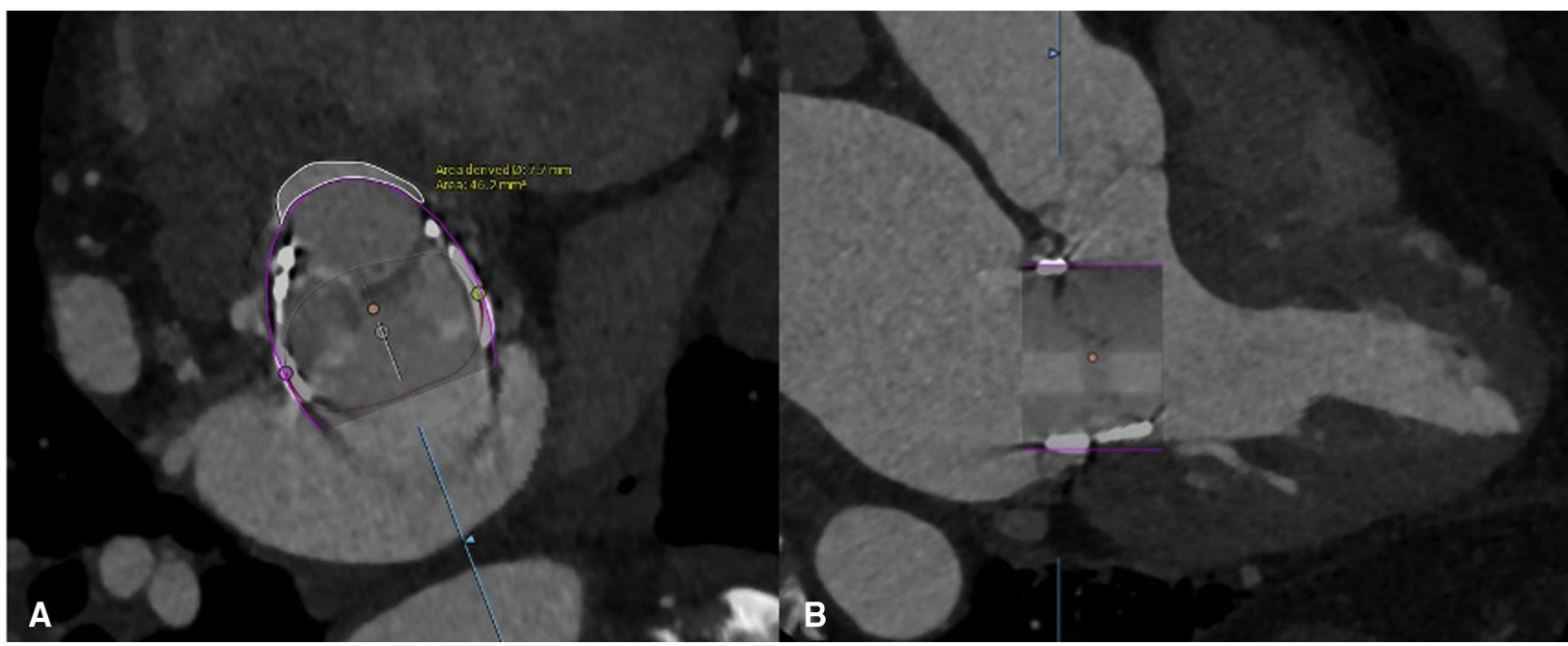

FIGURE 1. Preoperative computed tomography images with a simulated $29 \mathrm{~mm}$ valve-in-valve Edwards SAPIEN 3 transcatheter heart valve (Edwards Lifesciences, Irvine, Calif) in the mitral position demonstrating high risk for LVOT obstruction. A, The predicted neo-LVOT area was measured at $0.46 \mathrm{~cm}^{2}$. B, Valve-in-vale mitral valve replacement would have resulted in near-total obliteration of the neo-LVOT at end-systole.

Following median sternotomy, CPB was established with aortic and bicaval cannulation. After diastolic arrest, the mitral valve was exposed via Sondergaard's groove. Using a nerve hook pierced through the leaflets of the old bioprosthetic valve, we excised the 2 leaflets adjacent to the LVOT with a \#11 blade, thereby mitigating the risk of ViV-MVR induced LVOT obstruction (Video 1). Then, a

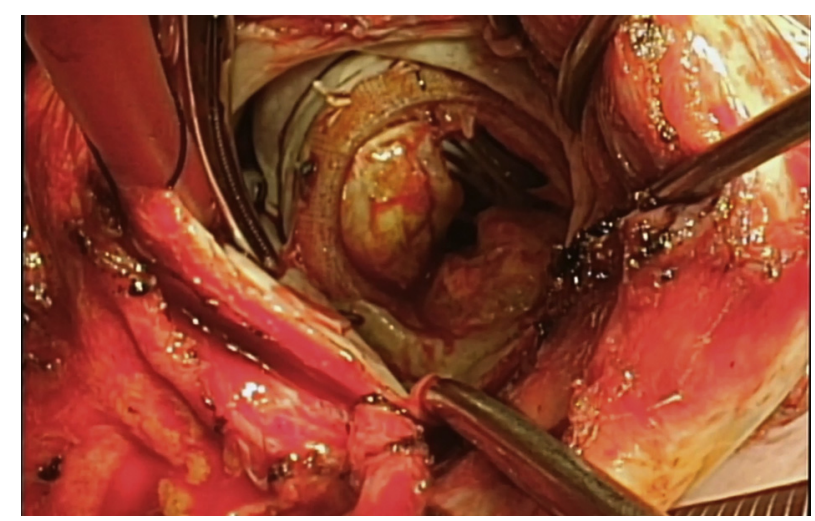

VIDEO 1. After establishing cardiopulmonary bypass and arresting the heart, the mitral valve was exposed through Sondergaard's groove. The 2 bioprosthetic leaflets of the indwelling 29-mm Edwards valve adjacent to the LVOT were excised with a \#11 blade. This maneuver was performed to mitigate the risk of LVOT obstruction after ViV TMVR deployment. A 29-mm SAPIEN 3 valve was introduced into the mitral valve annulus over a wire. Under direct vision, the balloon was slowly inflated until the valve was properly secured within the confines of the mitral annulus. Postprocedural saline test confirmed leaflet closure and valve competence. Bypass and crossclamp times were 60 and 35 minutes, respectively. Intraoperative TEE demonstrated successful mitral valve positioning with no paravalvular leak or significant gradient across the LVOT. Video available at: https://www.jtcvs.org/article/S2666-2507(20)30317-5/fulltext. 29-mm SAPIEN 3 valve was introduced into the mitral annulus over a wire to reduce the risk of left ventricular perforation. Under direct vision, the valve was advanced until the annular sealing cuff was just visible to ensure atrial positioning during deployment. The shaft of the delivery system was adjusted with forceps to ensure perpendicular orientation in relation to the deteriorated surgical valve. The balloon was slowly inflated until the transcatheter valve was secured within the ring of the old prosthesis. Postprocedural saline test confirmed leaflet closure and valve competence. CPB and crossclamp times were 60 and 35 minutes, respectively. Intraoperative transesophageal echocardiogram demonstrated satisfactory mitral valve positioning with no paravalvular leak or significant LVOT gradient.

The patient's postoperative course was uneventful, and she was discharged on postoperative day 8 . Transthoracic echocardiogram at 30-day follow-up showed a wellfunctioning bioprosthetic mitral valve with no paravalvular leak or LVOT obstruction (Figure 2). The mean gradient and peak velocity across the mitral valve were $5 \mathrm{~mm} \mathrm{Hg}$ and $1.1 \mathrm{~m} / \mathrm{s}$, respectively. The mean gradient across the LVOT was $7 \mathrm{~mm} \mathrm{Hg}$ with a maximum velocity of $1.3 \mathrm{~m} / \mathrm{s}$.

\section{DISCUSSION}

ViV-TMVR is a viable alternative for patients at elevated risk for repeat MVR. ${ }^{2}$ LVOT obstruction, however, is a feared complication associated with high mortality rates. ${ }^{3}$ Intentional transcatheter laceration of the anterior mitral valve leaflet to prevent LVOT obstruction (LAMPOON) with radiofrequency energy and transapical balloon-assisted translocation of the mitral anterior leaflet (BATMAN) techniques have proven feasible in preventing this problem but have yet to be replicated in larger series. ${ }^{4,5}$ The hybrid approach 


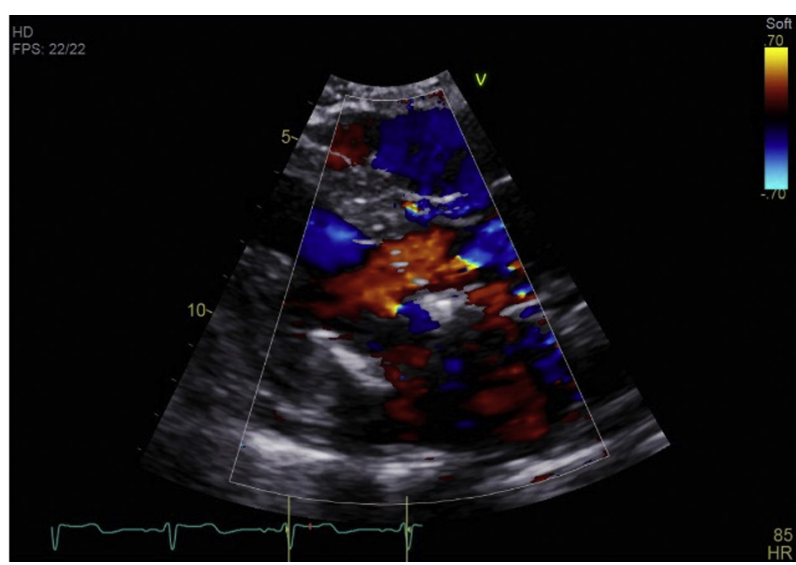

FIGURE 2. Postoperative transthoracic echocardiography demonstrating satisfactory flow through the LVOT and aortic valve at end-systole. The mean gradient and peak velocity across the mitral valve were $5 \mathrm{~mm} \mathrm{Hg}$ and $1.1 \mathrm{~m} / \mathrm{s}$, respectively. The mean gradient across the LVOT was $7 \mathrm{~mm}$ $\mathrm{Hg}$ and the maximum velocity was $1.3 \mathrm{~m} / \mathrm{s}$.

described herein takes advantage of transcatheter valve replacement with short $\mathrm{CPB}$ time while allowing for resection of bioprosthetic leaflets to minimize the danger of LVOT obstruction. This technique also obviates the need to resect the ring of an old bioprosthesis, potentially reducing the risk of permanent pacemaker placement or injury to the circumflex artery associated with repeat MVR. ${ }^{1} \mathrm{~A}$ limitation of the procedure is the need for $\mathrm{CPB}$, which may exclude applicability to extreme-risk patients. In conclusion, hybrid ViV-MVR adds another option to address the problem of LVOT obstruction in high-risk patients for whom repeat MVR is best avoided.

\section{References}

1. Ejiofor JI, Hirji SA, Ramirez-Del Val F, Norman AV, McGurk S, Aranki SF, et al. Outcomes of repeat mitral valve replacement in patients with prior mitral surgery: a benchmark for transcatheter approaches. J Thorac Cardiovasc Surg. 2018;156: 619-27.e1

2. Shivaraju A, Michel J, Frangieh AH, Ott I, Thilo C, Schunkert H, et al. Transcatheter aortic and mitral valve-in-valve implantation using the Edwards sapien 3 heart valve. J Am Heart Assoc. 2018;7:e007767.

3. Yoon SH, Bleiziffer S, Latib A, Eschenbach L, Ancona M, Vincent F, et al. Predictors of left ventricular outflow tract obstruction after transcatheter mitral valve replacement. JACC Cardiovasc Interv. 2019;12:182-93.

4. Khan JM, Babaliaros VC, Greenbaum AB, Foerst JR, Yazdani S, McCabe JM, et al. Anterior leaflet laceration to prevent ventricular outflow tract obstruction during transcatheter mitral valve replacement. J Am Coll Cardiol. 2019;73: 2521-34.

5. Helmy T, Hui DS, Smart S, Lim MJ, Lee R. Balloon assisted translocation of the mitral anterior leaflet to prevent left ventricular outflow obstruction (BATMAN): a novel technique for patients undergoing transcatheter mitral valve replacement. Catheter Cardiovasc Interv. 2020;95:840-8. 